\title{
Design and Development of Mobile Academic Platform in Universities
}

\author{
Yunju Changa, ${ }^{\text {, }}$, Lili Hong, Guang Su, Xuesong Leng \\ The University of Science and Technology Liaoning, Anshan 114051, China. \\ acyjlnk@163.com
}

Keywords: Internet +, Scientific research platform, Android app,Academic atmosphere .

\begin{abstract}
Under the background of the less connection between teachers and students in universities and the rapid development of the Internet, and according to the needs of students and teachers, the topic was to design and develop university research and academic platform prototype based on Android. First of all, the overall design and function of the APP Module were analyzed, and then the main XML code of the UI layout and Java code to achieve function modules were given. It was designed to explore the best combination point between the modern mobile communications technology and college students' participating in teachers' research, to promote scientific research between teachers and students done more frequently, and to promote a better academic atmosphere on campus, so that academic research in colleges can step into a new world taking the fast train of the mobile Internet.
\end{abstract}

\section{Introduction}

The cultivation of college students' scientific research ability is the teaching goal of cultivating scientific and technological talents in universities, the need of bringing up innovative talents, the important content of teaching reform. It is also the basis of further study and employment of students [1]. In order to achieve the goal of cultivating innovative talents, one of the qualities that teachers should possess is to lead undergraduates into task groups and scientific research platforms. However, students have little time to communicate with their teachers in addition to having some exchanges in class. Students know little about teachers' scientific research topics, and it is very difficult for them to participate in scientific research projects. Therefore, how to make more undergraduate students participate in scientific research topics is particularly important and urgent[2-4]. According to the investigation and analysis of the scientific and cultural quality of college students by Huaizhong Yang and others, it is concluded that the level of scientific research and the academic atmosphere on campus are important measures to measure the quality of a school. The strong academic atmosphere on campus can stimulate the academic competition consciousness and innovation consciousness of college students [5]. To build a bridge between teachers and students, to promote the formation of a strong academic atmosphere on campus, make more undergraduates participate in the academic research of teachers, the academic platform prototype was designed and developed based on the Android system used mostly by smart phones [6]. According to Zhou's research on collaborative innovation in universities, the platform also contributes to interdisciplinary cooperation and exchanges, promoting interdisciplinary integration, innovation and development [7].

\section{Function Analysis of Each Module in Client}

\subsection{Registration Login Module}

The platform needs a real name registration login, and provides both student and teacher registration entry.SharedPreferences is an Android data store that uses key-value to store sample configuration. The username and password will be saved in the Shared Preferences after the first APP login is started, and the username and password will be read automatically from the Shared Preferences in follow-up login. 


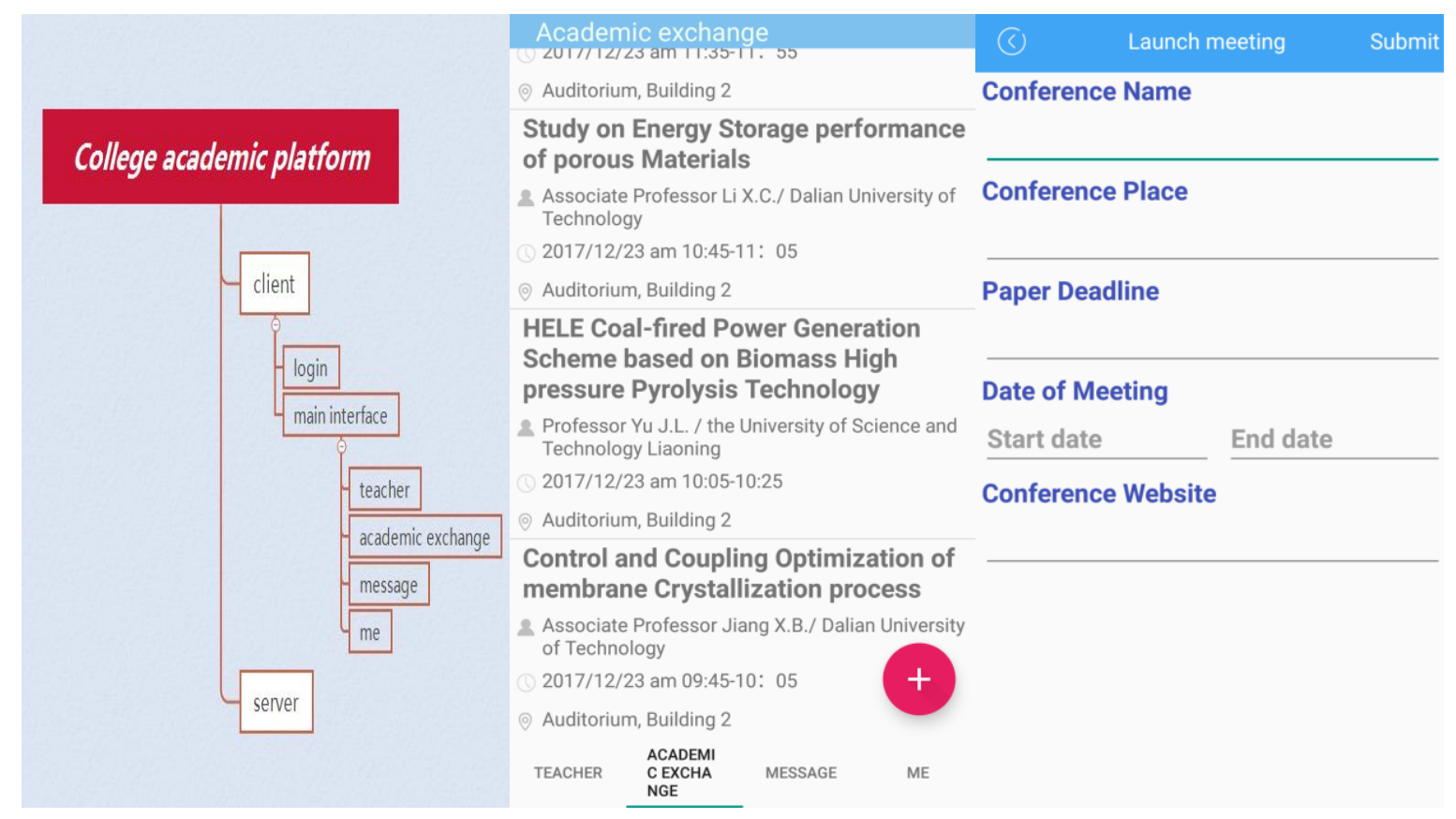

Fig 1. Design Framework Fig 2. Main interface Fig 3. Launch conference

Teacher registration needs to fill in the name, age, school, research field, scientific research experience and contact information. After teacher registration, personal information will be displayed in the teacher introduction module by family name ranking. Student registration needs to fill in the name, age, specialty class, interest, specialty and other personal introduction.

\subsection{Main Interface}

The main interface adopts the interface design pattern of APP's popular bottom navigation menu. The four main function modules of bottom navigation menus are respectively "TEACHER", "ACADEMIC EXCHANGE", "MESSAGE", "ME".

(1) Teacher module. It is used to show the field that teachers are engaged in, the research and the scientific research projects that are being carried out or will be carried out, so that students can have a better understanding of teachers' scientific research fields, research projects and choose the projects they are interested in and contact teachers to participate in scientific research.

(2) Academic exchange module. It is used to release academic conference and some academic salon activities on campus, the purpose of which is to make students know more about academic meeting and other information. All sponsored academic conference and other communication activities will be displayed in this module. Students can choose to enroll. In addition, to publish academic conferences in this module can save a certain meeting poster costs.

(3) Message module. Mainly used for private letters between students and teachers, mutual understanding and better selective cooperation. In addition, some system notifications will be pushed.

(4)"Me" module. This module can maintain the personal information filled out at the time of registration, in addition to checking for updates to app, contacting developers, making a personal configuration and exiting, etc.

\section{Platform Function Realization}

\subsection{Development Environment}

Development language: Java

Operating system platform: Microsoft Windows 10

Development tool: Android studio 2.2.2, jdk 1.8.0

\subsection{Android Development Environment}

Android system is a mobile phone system platform which was developed by Google and published in November 5th 2007. It is composed of five parts: Linux kernel, Linux native library, Android 
running environment, application framework and application program. It has strong fault-tolerant ability and security [9-11]. Through the following four installation configurations, the Android environment of developing academic and scientific research platform software in colleges and universities is built: JDK installation. $\rightarrow$ Android Studio installation. $\rightarrow$ Android SDK installation. $\rightarrow$ Create AVD.

\subsection{UI Layout for the Main Interface}

Android Studio recommend using the XML layout file to implement UI layout[12-13]. The main interface of app is implemented by Tabhost component and FrameLayout embedded ListView in the activity_main.xml file. The screenshot of main interface is shown in figure 2. The Tabhost component for the bottom navigation menu of the main interface is defined as follows:

$<$ TabHost

android="@android/tabhost"

android:layout_width="match_parent"

android:layout_height="match_parent"

android:layout_weight="1">

$</$ TabHost $>$

The framelayout is contained within the tabhost component and is defined as follows:

$<$ FrameLayout

android:id="@android:id/tabcontent"

android:layout_width="match_parent"

android:layout_height="match_parent"

android:layout_weight="1">

$</$ Framelayout $>$

The information of "TEACHER", "ACADEMIC EXCHANGE", "MESSAGE", "ME" module is displayed in the form of list with ListView weight, and the implementation of the information list of "ACADEMIC EXCHANGE" module is described in detail as following:

The "ACADEMIC EXCHANGE" module mainly displays topics, speakers/ unit, time, location information of academic conferences, reports, salons, etc. The style of each item unit in ListView is defined in the sch_item.xml file, in which topic, speakers/unit,time, location information is arranged in a Linearlayout from top to bottom.

The right corner uses the FAB suspending button, which is bound to the ListView list, and when clicked, it goes to the releasing conference interface, which is shown in figure 3.

\subsection{Implementation of the Main Interface Function}

Combining the UI layout above, the "ACADEMIC EXCHANGE" module of bottom navigation menu was achieved in the main interface function file MainAcivity.java.

(1) Implementation of bottom navigation menu

Step 1: Initialize the Tabhost component Tabhost code as follows: private TabHost tabHost.

Step 2: Get the Tabhost component Tabhost code as follows: tabHostn getTabHostCraft;

Step 3: Create the add bottom navigation menu as follows:

TabHost.TabSpec tab0 = tabHost.newTabSpec("tab1")

setIndicator("TEACHER").setContent(R.id.tab00);

tabHost.addTab(tab0);

TabHost.TabSpec tab1 = tabHost.newTabSpec("tab1")

setIndicator("ACADEMIC EXCHANGE") .setContent(R.id.tab01);

tabHost.addTab(tab1);

TabHost.TabSpec tab2 = tabHost.newTabSpec("tab2")

setIndicator("MESSAGE"..setContent(R.id.tab02);

tabHost.addTab(tab2);

TabHost.TabSpec tab3 = tabHost.newTabSpec("tab3")

setIndicator("ME").setContent(R.id.tab03);

tabHost.addTab(tab3); 


\section{(2)Function realization of "ACADEMIC EXCHANGE" module}

The "ACADEMIC EXCHANGE" module get the topic, speaker/ unit, time, location information of each conference is read from the database and respectively saved in four String arrasessch_titles,sch_authors_danwei,sch_times and sch_places,which are loaded by the corresponding weights in the sch_item.xml layout.

List $<$ Map $<$ String, Object $>>$ listitems_schoolar = new ArrayList $<$ Map $<$ String, Object $>>()$;

for (int $\mathrm{i}=0$; $\mathrm{i}<\mathrm{sch} \_$titles.length; $\left.\mathrm{i}++\right)\{$ //Adding elements to the list

Map $<$ String,Object $>$ listitems $=$ newHashMap $<$ String, Object $>()$;

listitem.put("sch_titles", sch_titles[i]);

listitem.put("sch_authors_danwei",ch_authors_danwei[i]);

listitem.put("sch_times", sch_times[i]);

listitem.put("sch_places", sch_places[i]);

listitems_schoolar.add(listitem);

\}

SimpleAdapter adapterSchoolar $=$ new

SimpleAdapter(this,listitems_schoolar,R.layout.schoolar,new String [ ]

\{ "sch_titles","sch_authors_danwei","sch_times","sch_places"\},

new int[]\{R.id.sch_titles,R.id.sch_authors_danwei,

R.id.sch_times,R.id.sch_places\}); //Create the adapter object

listviewSchoolar.setAdapter(adapterMessagesch);//Bind the adapter object to List View

\section{Conclusion}

The "Internet+" campus academic research platform is not only an upgrade of the intelligent campus information construction in colleges and universities, but also a higher education to meet the challenges of the mobile Internet era and a gesture of daring to seek change. Under the background of the rapid development of mobile Internet and the action plan of "Internet+", the prototype design and development of academic platform for scientific research in colleges and universities based on Android is carried out.The platform can not only help students have a deeper understanding of the teachers' scientific research fields and research topics, but also get to know more information such as the reports of academic conferences and so on. It can also serve as an online communication platform for teachers and students to promote the subject of teachers and students with more frequent research cooperation and the academic atmosphere on campus more intense. "Internet+" scientific research platform plays an important role, which can not be replaced by traditional scientific research platform. The academic platform aims to explore the best combination of modern mobile communication technology and college students' participation in teachers' scientific research, so that academic research in colleges can step into a new world taking the fast train of the mobile Internet.

\section{Acknowledgements}

This work was financially supported by the Liaoning College Students innovation and Entrepreneurship Program (201710146000042,201710146000083,201710146000206), University of Science and Technology Liaoning Teaching Reform Project (XJGYB201717) and University of Science and Technology Liaoning Experiment Teaching Reform Project.

\section{References}

[1]. Changbao Jiang,Discussion on the ways and methods of cultivating undergraduate's Scientific Research ability.Science and technology management research.2010,30(08):152-154.

[2]. Yanxiang He,IT Development and Innovation Talent training Computer Education .2009(03):9-14. 
[3]. Zhihan He,Ling Gan,Xinglian Chen,et al.Current situation, problems and countermeasures of Teacher-student Communication in Colleges and Universities: a case study of Southwest Jiaotong University.Journal of Southwest Jiaotong University (Social Sciences Edition).2014,15(05):111-115.

[4]. Weizhi Shao,Yaxuan Guo.A study on the current situation and training Mode of Master degree training in China.New West (second half of the month).2009(02):134+128.

[5]. Huaizhong Yang,Xueqing Chen,Shiyong Hu.Investigation and analysis of the scientific and cultural quality of contemporary college students.Journal of Wuhan University of Technology (Social Sciences Edition).2010,23(04):606-610.

[6]. Hongshen Wu,Jiangyang Li.Software Design of Android Aquatic Product Marketing system.Laboratory research and exploration.2016,35(07):149-153.

[7]. Jiliang Zhou.The Internal Realistic Dilemma and system improvement of University Cooperative Innovation.Journal of Sichuan normal University (Social Sciences Edition).2012,39(06):15-22.

[8]. Liang Yang,Song Yuan,Peng Jiang,et al.Research on Application of data Storage Mechanism based on Android platform.Computer knowledge and Technology.2014,10(11):2481-2484.

[9]. Lihui Yuan,Yi Huang,Simulation of Cloud Tourism oriented Real-time Management system based on android platform.Laboratory research and exploration.2014,33(10):50-54.

[10]. Di Zhang,Zheng Gong.Software Design of aircraft Paperless maintenance work order system based on Android.Laboratory research and exploration.2015,34(03):90-95.

[11]. Ming Hao.Mobile phone Anti-theft and Forensics system based on Android.Laboratory research and exploration.2014,33(09):33-36.

[12]. Ling Xie,Kun Lu,Qingqing Liu.Design and implementation of Campus Assistant based on Android.Laboratory research and exploration.,2017,36(07):144-147.

[13]. Pingping Bao,Guang Chen,Penghui Wang,et al.Design of vehicle Monitoring system based on Android platform..Laboratory research and exploration.2016,35(12):72-78. 